\title{
Holistic and analytic processes in the comparison of letters
}

\author{
DAVID A. TAYLOR \\ University of Rochester, Rochester, New York 14627
}

\begin{abstract}
College students made "same-different" judgments about successively presented pairs of letters whose degree of difference, in terms of the number of line segments out of register, was systematically varied. Reaction time was found to decrease with increases in the degree of difference in "different" responses, as well as to depend on the number of line segments present in the second (probe) letter. "Same" responses were much faster than different responses and were unaffected by the number of segments in the probe letter. This pattern of results suggests analytic (component-by-component) processing of "different" letters and holistic processing of "same" letters. The results have important implications for studies of multi-letter comparisons, in which it is commonly assumed that letter comparisons are holistic in nature.
\end{abstract}

In recent years, there has been considerable interest in the manner in which people execute mental comparisons. Typical paradigms used to investigate this issue are the popular memory-search task (Sternberg, 1966), the visual-search analog of this task (Atkinson, Holmgren, \& Juola, 1969), and the multiletter matching task (Bamber, 1969; see Nickerson, 1972, for a review of these types of task). The primary concern of studies using these paradigms has been with such questions as whether comparisons are executed one after the other (serially) or simultaneously (in parallel), and whether comparisons are halted when sufficient information has been obtained (self-terminated processing) or continued until all possible comparisons have been completed (exhaustive processing).

In order to ask such questions, of course, the investigators must first identify the unit of comparison. That is, he must know how his subjects chunk the information in the stimuli before he can determine the manner in which these chunks are processed. This logically prior issue has, however, been largely ignored in the research concerned with the character comparison process. Rather, most investigators have tacitly assumed that the unit of comparison was the same as the units that they varied in their experimental designs. For example, the three studies cited above each used letters and digits as the stimuli to be compared, and the models of the comparison process offered in the three reports all assumed that these characters represented the unit of comparison.

This assumption is convenient but questionable,

This research was supported by Grant No. GB-43913 from the National Science Foundation. I am indebted to Ralph Haber for the use of the apparatus described herein, and to Bob Cali, Paul Kleeberg, and Ron Shapiro for their assistance in the execution of the study. especially in view of the many studies of visual recognition which demonstrate the essential role of visual features in the identification of letters and digits (see Reed, 1973, for a review). The veracity of the assumption is important because an error in the identification of the unit of processing can invalidate the analysis of processing (Taylor, 1976a, pp. 183-185). For example, suppose that subjects performed a memory-search task by serially comparing the visual features of the probe against the features of the letters in the memory set, and that the subjects examined each of the memory letters for a given feature of the probe before turning to the next feature of the probe. The most competent analysis of processing, if based on the assumption that the letter was the unit of processing, could only lead to the conclusion that processing was parallel, even though no parallel operations were involved.

To my knowledge no one has yet made an experimental test of the possibility that subjects perform discrete acts of comparison on the visual components of letters. The most effective demonstration of multiple comparison processes within individual letters would be a replication of the typical findings of multiletter matching experiments in a task involving a single pair of letters. These findings are (a) for "different" responses, decreasing reaction time (RT) with increases in the degree of difference (measured in terms of the number of mismatching letters), and (b) for "same" responses, RTs which are much too fast to be described by any model which accounts for the "different" RTs (Bamber, 1969, 1972; Taylor, 1976b; see also Nickerson, 1972). This combination of results has led Bamber (1969) and others (e.g., Hock, 1973; Taylor, 1976b) to conjecture different processes for "same" and "different" responses, with "same" responses being emitted by a fast, holistic process and "different" responses 
being emitted by a slow, analytic process.

In order to test for the foregoing pattern of results in the matching of individual letters, I conducted a "same"'-"different" experiment using letters generated from a fixed set of line segments. Given these letters, I was able to systematically vary the similarity of two letters by having the letters differ in one, two, or three line segments. Although the shape and selection of the letters used was thus constrained, the letters were designed to look as much like printed (Gothic) letters as possible in order to maximize the generalizability of the results of the study to experiments using less constrained sets of letters.

\section{Subjects}

Thirty-nine students enrolled in an introductory psychology course served for $1 \mathrm{~h}$ each as part of a course requirement. Three subjects were eliminated from the data analysis for exceeding a predetermined error criterion, as described below.

\section{Apparatus}

The experiment was controlled by a Nova 1220 computer manufactured by Data General Corporation. Instructions and feedback regarding performance were presented to the subjects on a Lexiscope 2000 alphanumeric terminal (Lexicon, Inc.), and the subjects' responses were entered by pressing either of two marked keys located in the outer corners of the keyboard of this terminal. The stimuli were presented on a Tektronix 5103N oscilloscope with a P31 phosphor. The oscilloscope was mounted above and behind the alphanumeric terminal so that it was at about eye level for the subject and separated from him by a distance of about $100 \mathrm{~cm}$.

\section{Stimuli}

The letters used in the comparison task were formed from five basic line segments, as shown in Figure 1. All comparisons

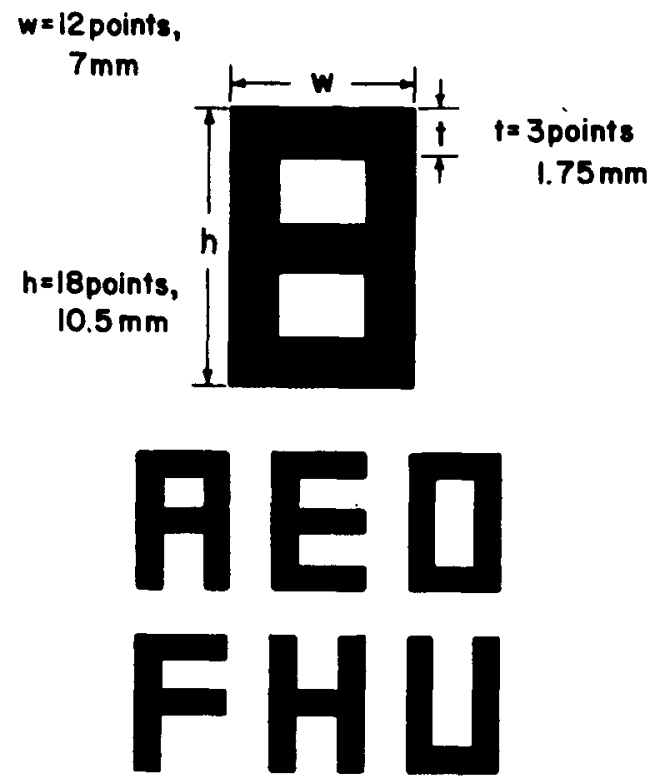

Figure 1. Format and selection of letters used in the experiment. involved the six letters shown in the figure. As can be seen, the six letters all contained the left vertical line segment but varied according to the presence or absence of the other four segments. Three of the letters contained three segments and three contained four segments. At $100 \mathrm{~cm}$, the distance between adjacent points in the display subtended $20 \mathrm{~min}$ of visual angle, so the letters were $4 \mathrm{deg} \times 6 \mathrm{deg}$ in size and the component line segments were 1 deg in width.

\section{Procedure}

Each trial consisted of the successive presentation of two letters, a criterion letter and a probe letter. Both letters appeared in the same location on the oscilloscope screen, being centered on a pair of cross hairs built into the screen. If the probe letter matched the criterion letter, the subject pressed a key that indicated "same"; if it did not, he pressed a key that indicated "different."

The time course of each trial was as follows: a 500 -msec presentation of the criterion letter, a 500 -msec interstimulus interval, a 500-msec presentation of the probe letter, and a 3-sec intertrial interval. Responses were accepted during a 1,500-msec interval measured from the onset of the probe letter. If a subject did not respond by the end of this interval, the message "TOO LATE" appeared briefly on the screen. Failures to respond during the allotted interval were treated as errors in subjects' feedback but were deleted from the data analysis reported below.

An experimental session consisted of two blocks of practice trials with 18 trials per block, followed by 10 experimental blocks of 36 trials each. Each experimental block consisted of 18 "same" trials and 18 "different" trials. On the "same" trials, a single letter appeared both as the criterion letter and as the probe, with each of the six letters in Figure 1 being used three times per block. The composition of the "different" trials is shown in Figure 2. Each of the six letters was used as the criterion letter three times per block, paired once with each of the probe letters shown in the right-hand side of the figure. As can be seen, the probe letters differed from the criterion letters in one, two, or three line segments. Because the changing of a single segment involved either the deletion or the addition of that segment, the way in which this change was made was balanced within each degree of difference (D). This balancing also caused the number of line segments present (three or four) to be balanced at each level of $D$. Within these constraints, a nearly optimal choice of letters was possible such that each letter appeared once at each level of D with but one exception; one instance of the letter $U$ (at $D=2$ ) was replaced with an instance of $H$.

Each practice block contained a random subset of the stimuli comprising a full experimental block. The data from the practice blocks were not included in the analyses reported below.

Each block began with the appearance of the message " $L O O K$ UP" on the Lexiscope screen. The word "READY" then appeared on the oscilloscope screen and the Lexiscope screen went blank. At the end of the block, the word "REST" appeared on the oscilloscope, and then this screen went blank and the Lexiscope displayed a table recording the number of errors and the mean RT for the block just completed. This table remained on the screen for $30 \mathrm{sec}$, and then it was replaced by the message "LOOK UP," which started the next block of trials.

Instructions for the task were presented on the Lexiscope screen, with the subject indicating comprehension of each panel of instructions by pressing a response key. The instructions included a general description of the task, a description of a typical trial, and an indication of which response key meant "same" and which meant "different." Half the subjects used their favored hands to make the "same" response, and half used their favored hands to make the "different" response. The instructions also described the nature of the feedback and directed the subjects to respond as quickly as possible without making more than two errors in any one block. Subjects who 


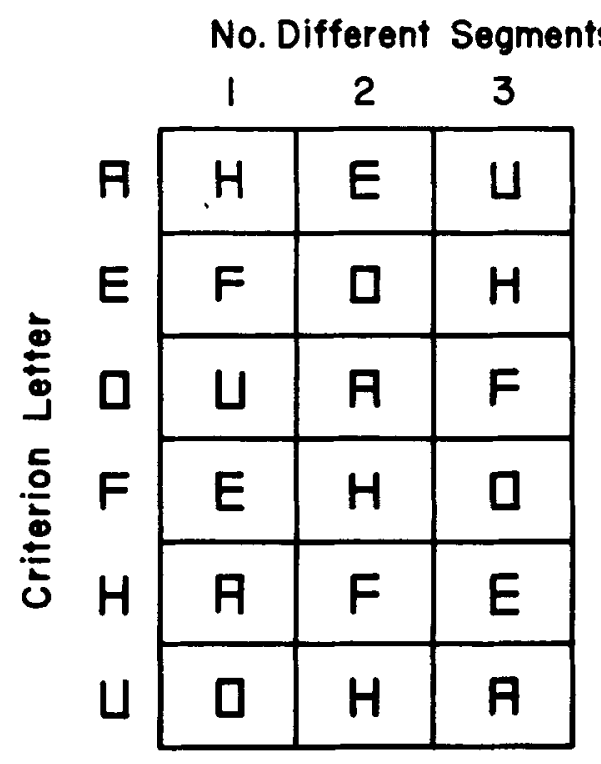

Figure 2. Composition of "different" trials.

made more than nine errors in any one block or who made an average of three or more errors in all the blocks (i.e., 30 or more errors for the entire session) were excluded from the data analysis. As noted above, three subjects were excluded for this reason, and their data were replaced by running additional subjects.

\section{RESULTS}

The mean RTs for correct responses and the mean proportions of errors are shown in Figure 3. Each point in this figure is based on either 1,080 observations (for "different" responses) or 3,240 observations (for "same" responses). There were only 10 failures to respond during the allotted 1,500 -msec interval out of a total of 12,960 observations, and these failures were excluded from consideration in the computation of the error rates.

The data in the figure are separated in terms of the number of line segments in the probe letter because that variable appeared to have a systematic influence on the speed and accuracy of responses. This influence also appeared in two pilot studies which preceded the present experiment, so it was not an unexpected finding.

Both the RT and error data were subjected to a one-way analysis of variance consisting of the following orthogonal components: (a) probe type ("same" vs. "different"), (b) number of line segments in the probe letter (three or four), (c) the interaction of the preceding influences, (d) the linear effect of $D$, the number of segments different between the criterion and probe letters, for "different" trials, (e) the quadratic effect of D for "different" trials, (f) the interaction between the linear effect of $D$ and number of segments, and $(\mathrm{g})$ the interaction between the quadratic effect of $D$ and number of segments.
The significant components for $\mathrm{RT}$ were probe type ("same" vs. "different"), $F(1,35)=89.72, p<.001$; number of segments in the probe letter, $F(1,35=4.43$, $\mathrm{p}<.05$; and the linear effect of $\mathrm{D}$ for "different" responses, $F(1,35)=39.53, p<.001$. There were no significant effects in the error data, although the linear effect of $D$ approached significance, $F(1,35)=$ 3.07 , calculated $\mathrm{p}=.087$.

The overall error rate was .0264 .

\section{DISCUSSION}

The pattern of results which typifies the multiletter matching task was well replicated in the present study using individual letters. That is, for "different" responses, RT decreased with increases in the number of different line segments, and "same" responses were considerably faster than "different" responses. Hence, to the extent that this pattern of results has been accepted as indicating holistic processing of "same" stimuli and analytic processing of "different" stimuli in studies using strings of letters, it must here be taken as evidence for holistic processing of matching letters and analytic processing of mismatching letters. This interpretation is bolstered by the small but reliable reduction in "different" $R$ Ts when the probe stimulus contained three rather than four line segments. It is not clear why such a reduction was obtained, since both the presence and the absence of segments communicated essential information (that is, subjects could not limit their comparisons to the segments present in the probe stimulus without making more errors than were observed). The presence of this effect in the "different" RTs, however, gives further evidence of analytic processing in "different" judgments, and the absence of the effect in "same" RTs gives further evidence of holistic processing in "same" judgments.

The lack of any significant effects in the error pro-

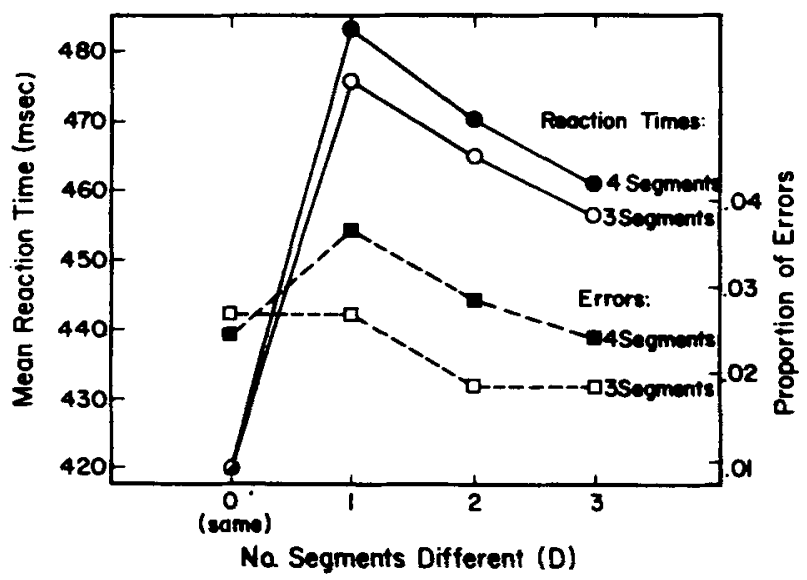

Figure 3. Mean RT and mean proportion of errors, plotted as a function of the number of line segments in the probe letter. 
portions indicates that none of the results just discussed was the product of a speed/accuracy tradeoff. For example, the greater speed of "same" responses reflects a genuine facilitation of processing for matching letters and not a response bias which decreased "same" RT at the expense of higher error rates. In fact, for the effects of $D$ and the number of features the error data follow a pattern very similar to the one observed for RTs. The failure of these apparent trends to reach significance may be due more to the small number of errors involved than to the validity of the effects in question.

In conclusion, the present study has demonstrated that, at least under some conditions, the act of comparing two letters consists of a number of comparisons on the visual components of those letters. Further research may be required to establish the generality of this finding, but the clear-cut results obtained in this one test certainly give reason for caution in the interpretation of experiments involving multiple comparisons among letters. Specifically, the possibility that the observed character comparisons actually consist of multiple feature comparisons must be entertained, with all the theoretical complications that this possibility entails. On a more positive note, the close correspondence between the results obtained with multiple letters and the results obtained for visual components of letters in the present study suggests that processing on the featural level is essentially similar to processing at the level of whole letters.

\section{REFERENCES}

Atrunson, R. C., Holmgren, J. E., \& Juola, J. F. Processing time as influenced by the number of elements in a visual display. Perception \& Psychophysics, 1969, 6, 321-326.

BAmber, D. Reaction times and error rates for "same" and "different" judgments of multidimensional stimuli. Perception \& Psychophysics, 1969, 6, 169-174.

BAMBER, D. Reaction times and error rates for judging nominal identity of letter strings. Penception \& Psychophysics, 1972, 12, 321-326.

Hock, H. S. The effects of stimulus structure and familiarity on same-different comparison. Perception \& Psychophysics, 1973. 14, 413-420.

Nickerson, R. S. Binary-classification time: A review of some studies of human information-processing capabilities. Psychonomic Monograph Supplements, 1972, 4(17, Whole No. 65).

REED, S. K. Psychological processes in pattern recognition. New York: Academic Press, 1973.

STERNBERG, S. High-speed scanning in human memory. Science, $1966,153,652-654$.

TAYLOR. D. A.. Stage analysis of reaction time. Psychological Bulletin, 1976, 83, 161-191. (a)

TAYLOR. D. A.. Effect of identity in the multi-letter matching task. Journal of Experimental Psychology: Human Perception and Performance, 1976, 2, 417-428. (b)

(Received for publication May 12, 1976; revision accepted July 13,1976 .) 